\title{
Elementos traqueais de cinco táxons de Cactaceae da caatinga pernambucana, Brasil
}

\author{
Emília Cristina Pereira de Arruda ${ }^{1,4}$, Marccus Alves² e Gladys Flávia Melo-de-Pinna $^{3}$
}

Recebido em 29/10/2003. Aceito em 24/03/2004

\begin{abstract}
RESUMO - (Elementos traqueais de cinco táxons de Cactaceae da caatinga pernambucana, Brasil). Este trabalho traz a caracterização morfológica de elementos de vaso e WBT (“traqueíde com espessamento largo”) de raiz e cladódio de cinco táxons de Cactaceae (Harrisia adscendens (Gurke) Britton \& Rose, Melocactus × horridus Wedermann Notizbl., M. zehntneri (Britton \& Rose) Luetzelb., Tacinga inamoena (Schumann) N.P. Taylor \& Stuppy e T. palmadora (Britton \& Rose) N.P. Taylor \& Stuppy) ocorrentes em um trecho de caatinga do Estado de Pernambuco, Brasil. Os elementos de vaso observados tanto nas raízes quanto nos cladódios apresentam-se praticamente idênticos entre os táxons estudados, sendo observadas pontoações alternas, opostas e escalariformes, além de placas de perfuração simples transversal e obliqua. As traqueídes (WBT) foram registradas apenas nos cladódios de quatro dos táxons estudados, exceto em Harrisia adscendens. As WBTs apresentam espessamento anelar ou helicoidal e o espessamento misto ocorre apenas em Melocactus. Os elementos traqueais foram medidos, mostrando que em Melocactus tanto a raiz como o cladódio, apresentam elementos de vaso longos e quanto as WBT essas são maiores no cladódio de Melocactus.
\end{abstract}

Palavras-chave: anatomia, Cactaceae, caatinga, elementos traqueais

ABSTRACT - (Traqueary elements of five taxons of Cactaceae of the caatinga from Pernambuco State, Brazil). This work brings a morphological characterization of traqueary elements (vessels and tracheids) in root and cladode of five taxons of Cactaceae (Harrisia adscendens (Gurke) Britton \& Rose, Melocactus $\times$ horridus Wedermann Notizbl., M. zehntneri (Britton \& Rose) Luetzelb., Tacinga inamoena (Schumann) N.P. Taylor \& Stuppy and T. palmadora (Britton \& Rose) N.P. Taylor \& Stuppy) occurent in the caatinga of Pernambuco State, Brazil. The vessels elements observed in the roots and cladodes seem almost same among the studied taxons, were observed alternate, opposite and scalariform pitting, and simple perforation plate transversal or oblique. The tracheids (WBT) were exclusively noted in the cladodes of four studied taxons, except Harrisia adscendens. The WBTs with annular and helical thickening are present in all taxons and the mix thickening is present only in Melocactus. The vessel elements and tracheids were measured and showing that the vessel elements are longer in roots and cladodes of Melocactus and the longest WBTs were only observed in cladode of Melocactus.

Key words: anatomy, Cactaceae, caatinga, traqueary elements

\section{Introdução}

O sistema vascular das Cactaceae encontra-se constituído basicamente por células condutoras do xilema e do floema como nos demais grupos de plantas vasculares. Porém em Cactaceae, geralmente, o tecido xilemático apresenta maior proporção de células parenquimáticas se comparadas à células de paredes lignificadas (elementos de vaso e fibras) compondo desta forma o chamado parênquima aqüífero (Soffiatti \& Angyalossy 2003; Mazzoni-Viveiros \& Costa 2003).

Alguns dos principais caracteres dos elementos de vaso das Cactaceae incluem pontoações escalariformes, alternas a irregulares e presença de placas de perfuração simples (Metcalfe \& Chalk 1950; Gibson 1973; 1977; Gibson \& Nobel 1986; Soffiatti \& Angyalossy 2003).

Além de elementos de vaso, fibras e células parenquimáticas, algumas espécies de Cactaceae podem apresentar no xilema, um tipo especial de traqueíde denominada "wide-band tracheid" - WBTs (Metcalfe \& Chalk 1950; Gibson 1973; Mauseth 1999). Este tipo de célula é caracterizado pela ausência de perfurações em suas paredes e presença de rígidas bandas de espessamento secundário, as quais podem ser anelares, helicoidais ou em dupla hélice, voltadas para o lume celular (Gibson 1977; Mauseth 1989; Landrum 2001).

\footnotetext{
1 Mestranda pela Universidade de São Paulo

2 Departamento de Botânica, Centro de Ciências Biológicas, Universidade Federal de Pernambuco, Av. Prof. Moraes Rego s/n, Cidade Universitária, CEP 50632-970, Recife, PE, Brasil

3 Instituto de Biociências, Universidade de São Paulo, C. Postal 4005, CEP 0161-970, São Paulo, SP, Brasil

4 Autor para correspondência: emilia.arruda@bol.com.br
} 
Ainda são poucos os trabalhos realizados com WBT, de maneira que sua origem e função ainda são incertas (Conde 1975), embora alguns pesquisadores relacionem sua ocorrência com a suculência (Boke 1944; Mauseth 1993). Porém, alguns trabalhos vêm sendo desenvolvidos para demonstrar que estas células podem ser importantes para as relações filogenéticas das Cactaceae (Mauseth et al. 1995; Mauseth \& Landrum 1997; Landrum 2002). Quanto às dimensões de elementos de vaso e fibras, alguns trabalhos foram desenvolvidos (Mauseth 1993; Mauseth \& PlemonsRodriguez 1998). Gibson \& Nobel (1986) mencionaram a possível correlação entre o comprimento e o diâmetro destas células com hábito das plantas.

O objetivo desse trabalho é caracterizar os elementos traqueais (elementos de vaso e WBT) de cinco táxons de Cactaceae ocorrentes em um trecho de caatinga do Estado de Pernambuco e, por meio destas observações, buscar detectar diferenças diagnósticas dessas células entre os órgãos (raiz e cladódio) bem como entre os táxons estudados.

\section{Material e métodos}

Este trabalho foi desenvolvido no Município de Alagoinha ( $08^{\circ} 29^{\prime \prime}$ S e $\left.36^{\circ} 47^{\prime \prime} \mathrm{W}\right)$, cerca de $780 \mathrm{~m} . \mathrm{s} . \mathrm{m}$. (FIAM 1994), nas Fazendas Cajueiro Seco e Lagoa Seca, localizadas em um trecho de caatinga do Estado de Pernambuco, no limite entre o Agreste e o Sertão Central, segundo a classificação proposta por AndradeLima (1957; 1960). Foram realizadas excursões à área de estudo para coleta dos órgãos vegetativos (raízes e cladódios) e reprodutivos dos táxons selecionados bem como observações de campo.

A seleção dos táxons foi efetuada por meio de consultas às coleções disponíveis nos herbários locais (Herbário da Universidade Federal de Pernambuco UFP; Herbário da Universidade Federal Rural de Pernambuco - PEUFR e Herbário da Empresa Pernambucana de Pesquisa Agropecuária - IPA). Devido à abundância na área de estudo, variedade de formas e pertencentes a diferentes subfamílias foram escolhidos os seguintes táxons: Harrisia adscendens (Gurke) Britton \& Rose, com hábito procumbente; Melocactus $\times$ horridus Wedermann Notizbl.; M. zehntneri (Britton \& Rose) Luetzelb., ambas com hábito globoso; Tacinga inamoena (Schumann) N.P. Taylor \& Stuppy e T. palmadora (Britton \& Rose) N.P. Taylor \& Stuppy ambas com hábito arbustivo. $H$. adscendens, Melocactus $\times$ horridus $\mathrm{e}$ $M$. zenhtneri são representantes da subfamília
Cactoideae, enquanto que Tacinga inamoena e T. palmadora da subfamília Opuntioideae (Hunt \& Taylor 1990). A classificação do hábito dos táxons estudados segue a proposta por Gibson \& Nobel (1986) para a família Cactaceae.

Após a coleta, parte do material foi destinado à confecção de exsicatas, segundo as normas usuais (Mori et al. 1989) e depositadas no Herbário UFP. Parte do material vegetativo (raízes e cladódios) coletado foi fixado em FAA 50 e, após 24h, transferido ao etanol 70\% (Johansen 1940), para sua preservação. A partir deste material, foi utilizada a técnica de dissociação, tanto das raízes como dos cladódios, com solução de peróxido de hidrogênio e ácido acético 1:1 (Franklin 1945). Após o material perder a coloração natural, o mesmo foi lavado em água destilada, até a completa remoção da solução de dissociação e acondicionado em etanol 70\% (Franklin 1945). Fragmentos deste material foram macerados sobre lâmina histológica, sendo adicionadas gotas de safranina $1 \%$ em etanol $50 \%$ (Kraus \& Arduin 1997) para a coloração e montados em glicerina 50\% (Purvis et al. 1964).

A análise dos elementos de vaso e das WBT foi realizada sob microscópio de luz. A mensuração foi efetuada em ocular milimetrada acoplada ao microscópio de luz. Para registro das células, as lâminas foram fotografadas em fotomicroscópio e as representações esquemáticas foram confeccionadas em câmara clara acoplada ao microscópio de luz.

A análise estatística dos resultados foi efetuada com auxílio do software BioEstat 2.0 (Ayres et al. 2000).

\section{Resultados}

Os elementos de vaso dos táxons analisados, tanto nas raízes como nos cladódios, caracterizam-se por apresentar placas de perfuração simples com posição transversal ou oblíqua, pontoações escalariformes, alternas e opostas e os apêndices também pontoados (Fig. 1A-C, 2A-U). Nas Figuras 3 e 4 é possível observar a variação no comprimento destas células, em raízes e cladódios, respectivamente.

Os testes estatísticos mostraram que elementos de vaso mais longos observados nas raízes pertencem a Melocactus, sendo esta diferença estatisticamente significativa $(\mathrm{p}<0,05)$ em quase todas as combinações realizadas, exceto entre $H$. adscendens e T. inamoena (Fig. 3). Nos cladódios, os maiores valores encontrados para os elementos de vaso ocorrem em $H$. adscendens 

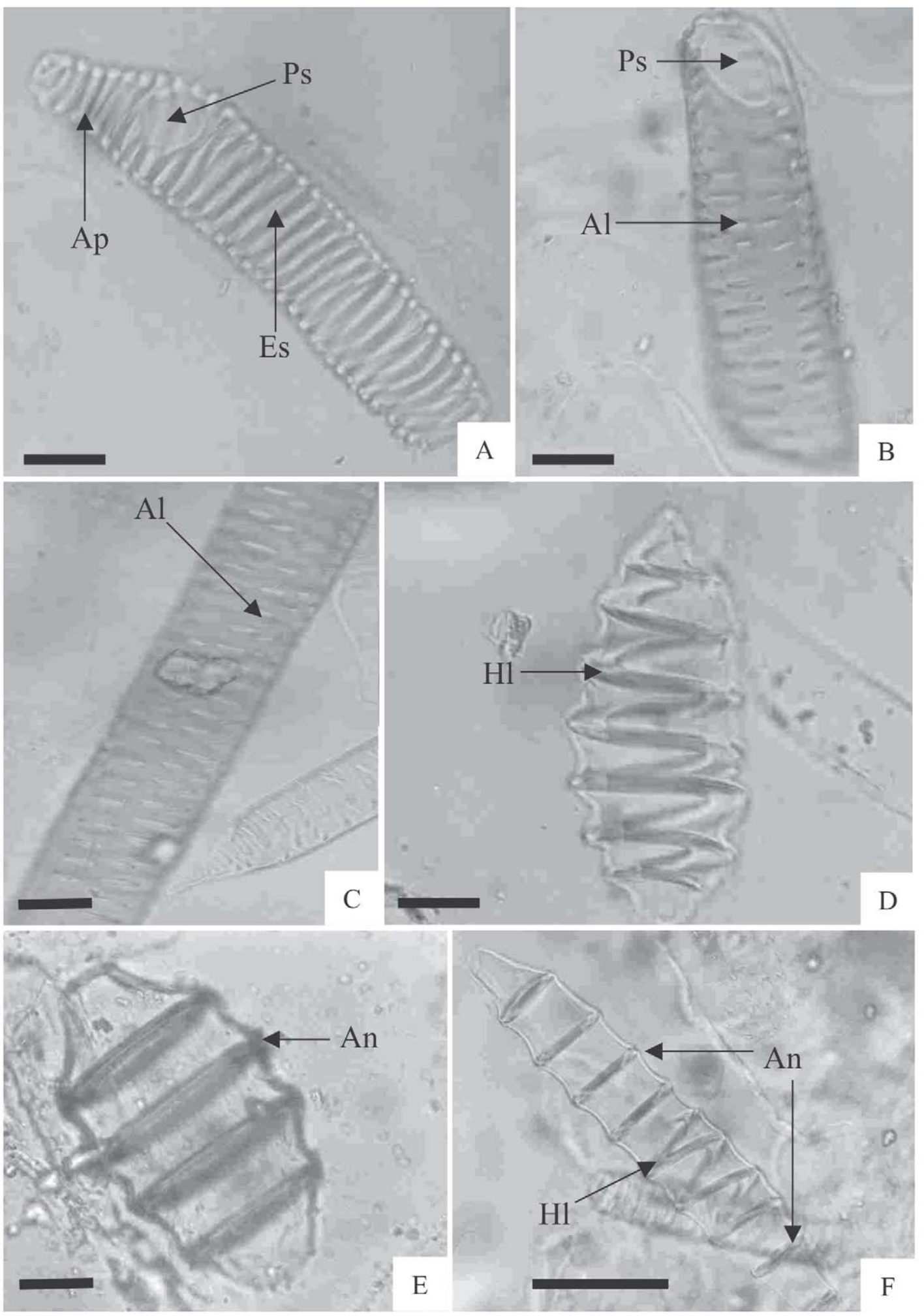

Figura 1. A-B. Elemento de vaso de raíz. A. Tacinga palmadora (Britton \& Rose) N.P. Taylor \& Stuppy evidenciando placa de perfuração simples e pontoações escalariformes. B. Melocactus $\times$ horridus Wedermann Notizbl. evidenciando placa de perfuração simples e pontoações alternas. C. Harrisia adscendens (Gurke) Britton \& Rose, elemento de vaso de cladódio evidenciando pontoações alternas. D-F. Melocactus, WBT. D. Melocactus zehntneri (Britonn \& Rose) Luetzelb., evidenciando espessamento helicoidal. E. Melocactus zehntneri, evidenciando espessamento helicoidal. F. Melocactus $\times$ horridus Wedermann Notizbl. evidenciando espessamento misto. Al - Pontoações alternas; An Espessamento anelar; Ap - Apêndice pontoado; Es - Pontoações escalariformes; Hl - Espessamento helicoidal; Ps - Placa de perfuração simples. Barra: A, B, D = 20 $\mu \mathrm{m} ; \mathrm{C}=15 \mu \mathrm{m} ; \mathrm{E}=25 \mu \mathrm{m} ; \mathrm{F}=30 \mu \mathrm{m}$. 


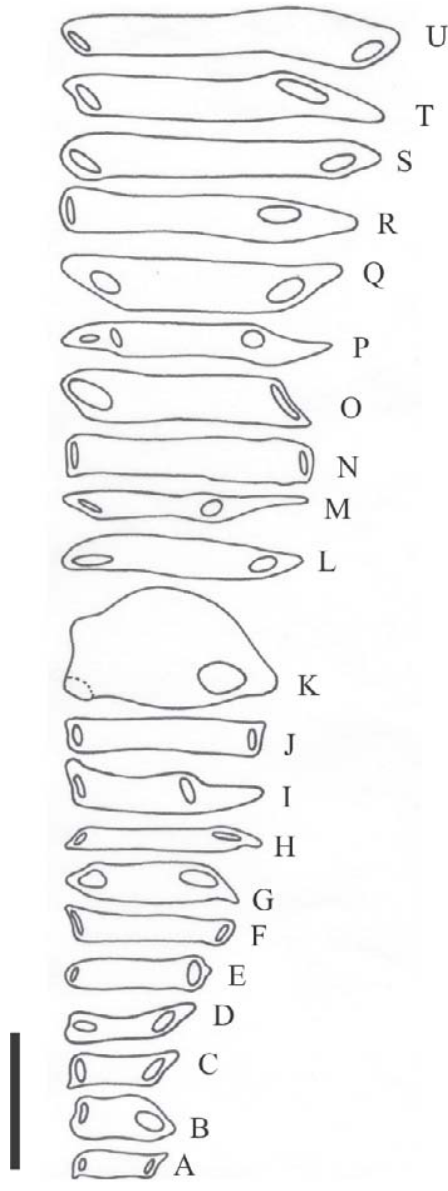

Figura 2. A-U. Representação esquemática dos principais tipos de elementos de vaso de raízes e cladódios de Cactaceae. A-C. Cladódio. A. Tacinga palmadora (Britton \& Rose). B. Melocactus $\times$ horridus Wedermann Notizbl. C. Tacinga inamoena (Schumann) N.P. Taylor \& Stuppy. D. Raiz, Harrisia adscendens (Gurke) Britton \& Rose. E-F. Cladódio. E. Tacinga inamoena. F. Tacinga palmadora. G-H. Raiz. G. Harrisia adscendens. H. Tacinga palmadora. I. Cladódio, Melocactus zehntneri (Britton \& Rose Luetzelb. J. Raiz, Harrisia adscendens. K-L. Cladódio. K. Melocactus $\times$ horridus. L. Melocactus zehntneri. M-N. Raiz. M. Tacinga palmadora. N. Tacinga inamoena. O-S. Cladódio, Melocactus zehntneri. P. Melocactus $\times$ horridus. Q. Harrisia adscendens. R. Melocactus zehntneri. S. Harrisia adscendens. T. Raiz, Melocactus $\times$ horridus. U. Cladódio, Harrisia adscendens. Barra $=150 \mu \mathrm{m}$.

e em Melocactus, sobretudo em M. zehntneri, sendo esta relação também estatisticamente significativa em quase todas as combinações realizadas, exceto entre M. × horridus e M. zehntneri (Fig. 4).

Apenas no xilema dos cladódios de Melocactus $\times$ horridus, M. zehntneri, Tacinga inamoena e T. palmadora, os quais são mais suculentos dentre os táxons estudados, foram observadas WBT; em Harrisia adscendens, o cladódio é menos suculento. As rígidas bandas de espessamento secundário, presentes nestas células, apresentam-se de três tipos: helicoidal (Fig. 1D), anelar (Fig. 1E) e misto (Fig. 1F), cujos espessamentos anelar e helicoidal ocorrem na mesma célula. As WBT com espessamento helicoidal e anelar foram observadas em Melocactus × horridus, M. zehntneri, Tacinga inamoena e T. palmadora, enquanto as com espessamento misto foram registradas apenas nos cladódios de Melocactus. É possível notar ainda variações quanto

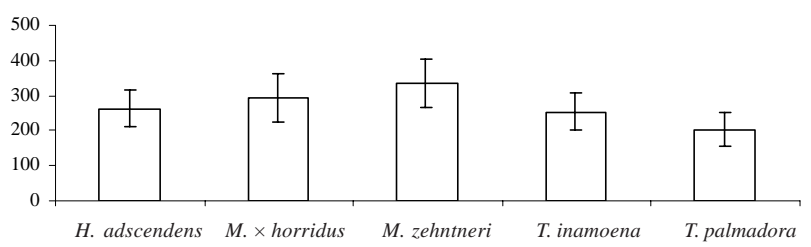

Figura 3. Variações do comprimento $(\mu \mathrm{m})$ dos elementos de vaso na raiz dos cinco táxons estudados, pertencentes aos gêneros Harrisia, Melocactus e Tacinga.

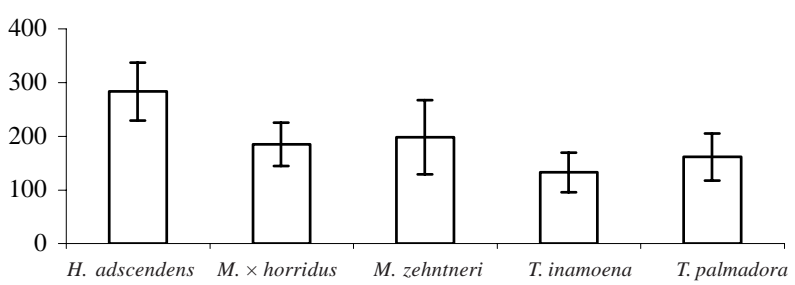

Figura 4. Variações do comprimento $(\mu \mathrm{m})$ dos elementos de vaso no cladódio dos cinco táxons estudados, pertencentes aos gêneros Harrisia, Melocactus e Tacinga.

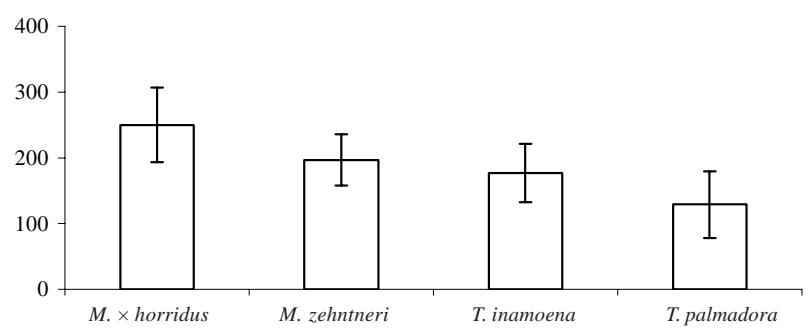

Figura 5. Traqueides com espessamento largo - WBT ("wideband tracheids”) no cladódio: variações do comprimento ( $\mu \mathrm{m})$ em quatro táxons pertencentes aos gêneros Tacinga e Melocactus.

ao comprimento das WBT entre os táxons estudados (Fig. 5), sendo que as mais longas ocorrem em Melocactus, principalmente em $M . \times$ horridus. Esta relação é estatisticamente significativa para todas as combinações realizadas. 


\section{Discussão}

Os elementos de vaso dos cladódios e raízes dos táxons analisados apresentam-se basicamente como descritos na literatura das Cactaceae (Metcalfe \& Chalk 1950; Gibson 1977; Soffiatti \& Angyalossy 2003), não havendo especificidade de tipos entre os táxons estudados.

Quanto aos padrões dimensionais, foi observado que o comprimento dos elementos de vaso dos táxons analisados neste trabalho, está dentro dos valores esperados para as Cactaceae $(100-500 \mu \mathrm{m})$ como mencionado por Metcalfe \& Chalk (1950).

Gibson \& Nobel (1986), por meio de análise em 119 espécies de 50 gêneros da subfamília Cactoideae e 35 espécies de 17 grupos de Platyopuntias, constataram a correlação entre os comprimentos dos elementos de vaso e de fibras com o porte da planta, sendo estas células mais longas quanto maior o porte da planta. Entretanto, Carlquist (1975) e Zimmerman (1983) evidenciaram a ocorrência de elementos de vaso mais curtos em espécies arbustivas de regiões áridas, como é encontrado na maioria das Cactaceae. Nos táxons estudados não parece haver correlação entre o comprimento dos elementos de vaso e o porte das plantas, tendo-se em vista uma tendência destas células serem mais longas, tanto em raízes como em cladódios, em Melocactus, as menores plantas analisadas. Embora $H$. adscendens, planta de maior porte em estudo, apresente elementos de vaso mais longos nos cladódios, é possível notar padrão similar em $M$. zehntneri.

Conde (1975) evidenciou comprimento dos elementos de vaso relativamente constante nas cinco espécies de Opuntia por ele analisadas. Segundo o autor, isto pode ser devido à relativa similaridade entre as espécies. Este aspecto pode, em parte, ser corroborado no presente estudo, quando comparados os dois táxons de Tacinga (Tacinga inamoena $132 \mu \mathrm{m}$ e T. palmadora $-161 \mu \mathrm{m})$, bem como os dois de Melocactus $(M$. × horridus - $185 \mu \mathrm{m}$ e M. zehntneri - $198 \mu \mathrm{m})$, embora o número de representantes de cada gênero seja pequeno.

Bailey (1957) mencionou tendência evolutiva geral de redução do comprimento dos elementos de vaso e das fibras em Cactaceae. Com base neste caráter as plantas do presente estudo, que seriam mais derivadas, as Tacinga, são na realidade consideradas menos derivadas do que os Melocactus (Hunt \& Taylor 1990), os quais apresentam elementos de vaso mais longos. Segundo Mauseth (1993), elementos de vaso curtos evitam o embolismo e a conseqüente interrupção na condução, sobretudo em plantas de ambientes secos, como as aqui estudadas.

A ocorrência de WBT em Cactaceae é mencionada por vários autores (Metcalfe \& Chalk 1950; Gibson \& Nobel 1986; Mauseth 1999; Landrum 2002). A função e origem destas células ainda são incertas (Conde 1975), embora alguns autores correlacionem sua ocorrência com o grau de suculência da planta (Boke 1944; Gibson 1977; Fahn \& Cutler 1992; Mauseth 1993), enquanto outros com a evolução da mesma (Mauseth et al. 1995; Landrum 2002). Segundo Gibson (1977), quando estas células estão presentes há tendência tanto à redução da proporção de elementos de vaso, quanto à ausência de fibras no xilema. Embora as WBT tenham permitido a distinção dos táxons analisados, tendo-se em vista sua ausência unicamente em $H$. adscendens, as afirmações feitas pelos autores acima citados sugerem que estudos devem ser realizados nestes e em outros táxons para comprovar a real função destas estruturas.

Devido às WBT ocorrerem em tecidos excessivamente suculentos, a possível função das rígidas bandas de espessamento secundário é o fortalecimento deste tecido, auxiliando na sustentação (Bailey 1966, apud Conde 1975). Segundo Mauseth et al. (1995), estas bandas impedem o colapso das paredes primárias da célula mantendo o lume apto a conduzir mesmo em períodos de estresse hídrico. Ainda de acordo com o autor, devido a estas propriedades, as WBT podem representar importantes adaptações para plantas suculentas em ambientes xéricos. Os espessamentos ocorrentes nestas células podem ser anelares, helicoidais ou em dupla-hélice (Metcalfe \& Chalk 1950; Gibson \& Nobel 1986; Landrum 2001). Gibson (1977) mencionou a ocorrência de WBT tipicamente estreitas e fusiformes e com espessamento helicoidal em Cactoideae, embora o espessamento anelar possa ocorrer em representantes de pequeno porte da referida subfamília. Já as Opuntioideae, embora apresentem WBT estreitas e fusiformes, o espessamento destas células nestas espécies é anelar ou, raramente, anelar-helicoidal. Não há especificidade entre os tipos de espessamento das WBT dentre os táxons estudados, pois todos apresentam espessamento anelar e helicoidal, exceto quanto ao anelar-helicoidal, descrito aqui como misto, o qual ocorre unicamente em Melocactus.

Com base nos resultados obtidos é possível indicar que os elementos traqueais aqui observados corroboram as referências sobre formas e tipos descritos para as Cactaceae. Devido à similaridade destes elementos em 
raízes e cladódios, bem como entre os táxons estudados, não é possível a diagnose dos mesmos com base neste caráter. Como as WBT estão ausentes nas raízes de todos os táxons estudados e no cladódio de H. adscendens, estas podem representar um caráter diagnóstico ou, ainda estarem relacionadas com a suculência. Quanto ao padrão de espessamento, o tipo misto restrito aos cladódios de Melocactus também permite a diagnose dos táxons estudados.

Contudo, deve ser ressaltado que o reduzido número de táxons analisados, torna necessária a ampliação de estudos sobre os elementos traqueais em outros representantes da família, visando fixar os caracteres observados nestes táxons, além de estabelecer outros também úteis à diagnose.

\section{Agradecimentos}

Os autores agradecem ao Dr. Nigel Taylor, pela gentileza na identificação do material botânico; ao PIBIC/CNPq/UFPE (Proc. 20310), pela concessão e renovação da bolsa de iniciação científica concedidas à primeira Autora.

\section{Referências bibliográficas}

Andrade-Lima, D. 1957. Estudos fitogeográficos de Pernambuco. Recife, IPA.

Andrade-Lima, D. 1960. Estudos fitogeográficos de Pernambuco. Revista Arquivo do Instituto de Pesquisa Agronômica 5: 305-341.

Ayres, M.; Ayres Jr., M.; Ayres, D. \& Santos, A. 2000. BioEstat 2.0: Aplicações estatísticas nas áreas de ciências biológicas e médicas. Brasília, Sociedade Civil Mamirauá e CNPq.

Bailey, I.W. 1957. The potentialities and limitation of wood anatomy in the study of the phylogeny and classification of Angiosperms. Journal of the Arnold Arboretum 38: 243-254.

Boke, N.H. 1944. Histogenesis of the leaf and areole in Opuntia cylindrica. American Journal of Botany 31(6): 299-316.

Carlquist, S. 1975. Ecological strategies of xylem evolution. Berkeley, University California Press.

Conde, L. 1975. Anatomical comparisons of five species of Opuntia (Cactaceae). Annals of the Missouri Botanical Garden 62: 425-473.

Fahn, A. \& Cutler, D. 1992. Xerophytes. BerlinGebrüder Borntraeger.

FIAM. Fundação de Desenvolvimento Municipal do Interior de Pernambuco. 1994. Perfil Municipal do Interior de Pernambuco. Recife, PE.
Franklin, G. 1945. Preparation of thin sections of synthetic resins and wood - resin composites and a new macerating method for wood. Nature 155(39): 51.

Gibson, A. 1973. Comparative anatomy of secundary xylem in Cactoideae (Cactaceae). Biotropica 5(1): 29-65.

Gibson, A. 1977. Wood anatomy of Opuntias with cylindrical to globular stems. Botanical Gazette 138(3): 334-351.

Gibson, A. \& Nobel, P. 1986. The cactus primer. Cambridge, Havard University Press.

Hunt, D. \& Taylor, N. 1990. The genera of Cactaceae: progress towards consensus. Bradleya 8: 85-107.

Johansen, D. 1940. Plant microtechnique. New York, McGraw-Hill Book Co. Inc.

Kraus, J. \& Arduin, M. 1997. Manual básico de métodos em morfologia vegetal. Rio de Janeiro, EDUR.

Landrum, J.V. 2001. Wide-band tracheids in leaves of genera in Aizoaceae: the systematic occurence of a novel cell type and its implications for the monophyly of the subfamily Ruschioideae. Plant Systematics and Evolution 277: 46-61.

Landrum, J.V. 2002. Four succulent families and 40 million years of evolution and adaptation to xeric environments: What can stem and leaf anatomical characters tell us about their phylogeny? Taxon 51: 463-473.

Mauseth, J. 1989. Comparative structure-function studies within a strongly dimorphic plant, Melocactus inortus (Cactaceae). Bradleya 7: 1-12.

Mauseth, J. 1993. Medulary bundles and the evolution of cacti. American Journal of Botany 80: 928-932.

Mauseth, J. 1999. Comparative anatomy of Espostoa, Pseudoespostoa, Thrixanthocereus and Vatricania. Bradleya 17: 27-37.

Mauseth, J.D. \& Landrum, J.V. 1997. Relictual vegetative anatomical characters in Cactaceae: the genus Pereskia. Journal of Plant Research 110: 55-64.

Mauseth, J.D. \& Plemons-Rodriguez, B.J. 1998. Evolution of extreme xeromorphic characters in wood: a study of nine evolutionary lines in Cactaceae. American Journal of Botany 85(2): 209-218.

Mauseth, J.D.; Uozumi, Y.; Plemons, B.J. \& Landrum, J.V. 1995. Structural and systematic study of an ununsual tracheid type in cacti. Journal of Plant Research 108: 517-526.

Mazzoni-Viveiros, S. \& Costa, C. 2003. Periderme. In: Anatomia vegetal. B. Appezzato-da-Glória \& S. CarmelloGuerreiro. Viçosa, Ed. UFV.

Metcalfe, C. \& Chalk, L. 1950. Anatomy of the Dicotyledons. v.II. Oxford, Claredon Press.

Mori, S.A.; Silva, L.A.M.; Lisboa, G. \& Coradin, L. 1989. Manual de manejo de herbário fanerogâmico. Ilhéus, CEPLAC.

Purvis, M.; Collier, D. \& Walls, D. 1964. Laboratory techniques in botany. London, Butterworths.

Soffiatti, P. \& Angyalossy, V. 2003. Stem anatomy of Cipocereus (Cactaceae). Bradleya 21: 39-48.

Zimmerman, M. 1983. Xylem structure and the ascent of sap. New York, Springer, Berlin Heidelberg. 